\title{
9. Humanitäre Hilfe
}

Xavier Tschumi Canosa

\section{(2) OpenEdition \\ 1 Journals}

Electronic version

URL: http://journals.openedition.org/sjep/850

DOI: $10.4000 /$ sjep. 850

ISSN: 1663-9677

Publisher

Institut de hautes études internationales et du développement

\section{Printed version}

Date of publication: 1 avril 2001

Number of pages: 377-385

ISSN: 1660-5926

\section{Electronic reference}

Xavier Tschumi Canosa, «9. Humanitäre Hilfe », Schweizerisches Jahrbuch für Entwicklungspolitik [Online], 20 | 2001, Online erschienen am: 03 September 2012, abgerufen am 08 September 2020. URL : http://journals.openedition.org/sjep/850 ; DOI : https://doi.org/10.4000/sjep.850 


\section{HUMANITÄRE HILFE}

D IE HUMANITÄRE HILFE der Schweiz erreichte 1999 mit rund 400 Millionen Franken einen sehr hohen Betrag. Drei Viertel der Hilfe wurden von der DEZA aufgebracht, der Rest von anderen eidgenössischen Departementen sowie von den Kantonen und Gemeinden. Allein in den Balkan sind über 88 Millionen Franken (DEZA-Beiträge) geflossen, gefolgt von Afrika (mit über 59 Millionen), Asien (mit über 35 Millionen) und Lateinamerika (mit 15,5 Millionen).

Der ordentliche Beitrag an das Sitzbudget des Internationalen Komitees vom Roten Kreuz (IKRK) belief sich 1999 auf 66 Millionen Franken. Die multilateralen Aufwendungen betrugen rund 22 Millionen Franken, davon wurden 13 Millionen für das Hochkommissariat der Vereinten Nationen für Flüchtlinge (UNHCR) erbracht.

Die wichtigsten Aktionen der Schweiz für humanitäre Hilfe im Jahr 2000 wurden im Kosovo, in Afghanistan und in Mosambik durchgeführt.

Der neue Leiter der Abteilung Humanitäre Hilfe und SKH bei der DEZA wurde vom Bundesrat am 4. Dezember 2000 ernannt. Toni Frisch wird Charles Raedersdorf, dessen Stellvertreter er derzeit ist, am 1. Juni 2001 ablösen.

\subsection{KURZER ÜBERBLICK ÜBER DIE HUMANITÄRE HILFE DER SCHWEIZ}

Für die humanitäre Hilfe innerhalb der Bundesverwaltung ist ausschliesslich die DEZA zuständig. Die Umsetzung erfolgt durch ihre Abteilung Humanitäre Hilfe und das Schweizerische Katastrophenhilfekorps (SKH). Die humanitäre Hilfe wird durch mehrjährige Rahmenkredite finanziert, die dem Parlament vom Bundesrat unterbreitet werden. Sie dienen als Rahmen für die Beschlüsse über die jährlichen Budgetzuweisungen. Der letzte Rahmenkredit für humanitäre Hilfe wurde vom Parlament 1997 bewilligt und belief sich auf 1,05 Milliarden Franken $^{1}$. Er umfasst die Einsätze in den Ländern des Südens und den Oststaaten sowie die multilateralen Aufwendungen. Die ordentlichen Beiträge an das Sitzbudget des IKRK in Genf werden nicht aus diesem Rahmenkredit finanziert, sondern aus einem spezifischen Rahmenkredit, der ebenfalls 1997 erneuert wurde (65 Millionen Franken pro Jahr, von 1998 bis 2001)².

Die DEZA hat zehn Schwerpunktländer oder -regionen für ihre humanitäre Hilfe festgelegt. Es handelt sich um Mittelamerika, Kolumbien und Venezuela, Westafrika, Angola, das Gebiet der Grossen Seen, das Horn Afrikas, Palästina, Afghanistan, den Kaukasus und den Balkan (Stand vom 19. Dezember 2000).

1. Botschaft über die Weiterführung der internationalen humanitären Hilfe der Eidgenossenschaft vom 20. November 1996 (Botschaft Nr. 96.092).

2. Botschaft über die Finanzhilfe des Bundes an das Sitzbudget des Internationalen Komitees vom Roten Kreuz (1998-2001) vom 2. Juni 1997 (Botschaft Nr. 97.047). 
Wenn die DEZA für die humanitäre Hilfe auf Bundesebene zuständig ist, so können aber auch andere eidgenössische Departemente gewisse Aspekte dieser Hilfe finanzieren. Zudem bringen die Kantone und Gemeinden, wie auch die schweizerischen Nichtregierungsorganisationen aus ihren Eigenmitteln, Beiträge für die humanitäre Hilfe auf.

Die Einsatzbereiche der schweizerischen humanitären Hilfe sind Prävention, Rettung, Überleben und Wiederaufbau. Bei den humanitären Einsätzen verfügt die DEZA über vier verschiedene Instrumente: Entsendung von Personal (Angehörige des SKH), finanzielle Beiträge, Nahrungsmittelhilfe und Materiallieferungen. Die DEZA führt direkte Einsätze durch (zumeist durch das SKH, manchmal aber auch durch die Schweizer Botschaften oder die DEZA-Koordinationsbüros) und erbringt Beiträge an die internationalen Organisationen und die schweizerischen Hilfswerke. Bei ihren Einsätzen bedient sich die DEZA der vier oben genannten Instrumente.

\subsection{AUFWENDUNGEN FÜR HUMANITÄRE HILFE 1999}

Die humanitäre Hilfe der Schweiz belief sich 1999 auf über 398 Millionen Franken, wovon der grösste Teil von der DEZA aufgebracht wurde.

Tabelle 23: Öffentliche Entwicklungshilfeleistungen der Schweiz für die humanitäre Hilfe 1999, nach Finanzierungsquellen

\begin{tabular}{lrrr}
\hline & $\begin{array}{r}\text { Bilaterale } \\
\text { Zahlungen }\end{array}$ & $\begin{array}{r}\text { Multilaterale } \\
\text { Zahlungen }\end{array}$ & Gesamtbetrag \\
\hline DEZA & 281926816 & 21845565 & 303772381 \\
\hline Andere eidgenössische Departemente & 90407249 & 0 & 90407249 \\
\hline Kantone und Gemeinden & 4089560 & 0 & 4089560 \\
\hline Gesamtbetrag & $\mathbf{3 7 6 4 2 3 6 2 5}$ & $\mathbf{2 1 8 4 5 5 6 5}$ & $\mathbf{3 9 8 2 6 9 1 9 0}$ \\
\hline
\end{tabular}

Quellen: DEZA, Internationale Zusammenarbeit der Schweiz. Jahresbericht 1999. Humanitäre Hilfe, S. 10-11.

DEZA, Statistischer Dienst, Dezember 2000.

Ausserdem hat die DEZA noch rund 5 Millionen Franken für humanitäre Hilfe in den Transitionsländern des Ostens (hauptsächlich in Russland) aufgebracht. Diese Zahlungen können nicht als öffentliche Entwicklungshilfe, sondern nur als öffentliche Hilfe verbucht werden.

\section{$\square$ Instrumente und Einsatzformen der humanitären Hilfe}

Die direkten Einsätze des Schweizerischen Katastrophenhilfekorps (SKH) haben sich zwischen 1998 und 1999 im Wert fast verdreifacht und stiegen von 27,6 Millionen Franken auf 75 Millionen Franken an. 1999 hat das SKH 474 Einsätze in 38 verschiedenen Ländern der Erde durchgeführt. Im Balkangebiet (Kosovo, Jugoslawien, Albanien, Mazedonien und Bosnien-Herzegowina) hat das SKH seine Einsätze hauptsächlich auf die Hilfe für die Flüchtlinge konzentriert. Jedoch haben auch die Erdbeben in Taiwan, Griechenland und in der Türkei sowie die Kriegs- oder Nachkriegssituationen in Afrika einen bedeutenden Personal- und Materialeinsatz des SKH mobilisiert. Die Folgen des Wirbelsturms «Mitch» in Lateinamerika erfordern weiterhin ein grosses Engagement des SKH in dieser Region

凹 JSDW 2000, S. 342-343. 
Die Beiträge der DEZA an die internationalen Organisationen und die schweizerischen Hilfswerke beliefen sich 1999 auf über 229 Millionen Franken. Die internationalen Organisationen erhielten über 89 Millionen Franken in Form von ordentlichen Beiträgen (39 Millionen), spezifischen Beiträgen (30 Millionen) und Nahrungsmittelhilfe (20 Millionen). Das Hochkommissariat der Vereinten Nationen für Flüchtlinge (UNHCR) erhielt mit über 36 Millionen Franken den grössten Anteil der Beiträge. Ihm folgten das Hilfswerk der Vereinten Nationen für die Palästinaflüchtlinge im Nahen Osten (UNRWA) mit über 8 Millionen Franken, das UN-Büro für die Koordination humanitärer Angelegenheiten (OCHA) mit über 2,5 Millionen und die Internationale Organisation für Migration (IOM) mit 1,5 Millionen Franken. Somit dienen die multilateralen Beiträge der humanitären Hilfe der Schweiz in erster Linie zur Finanzierung humanitärer Aktivitäten im Zusammenhang mit den Bevölkerungsbewegungen.

1999 erhielt das IKRK 66 Millionen Franken an ordentlichen Beiträgen, zu denen noch über 27 Millionen für spezifische Beiträge kommen.

Die schweizerischen Nichtregierungsorganisationen haben 199942 Millionen Franken für humanitäre Hilfe erhalten, davon 30 Millionen in Form von spezifischen Beiträgen und den Rest in Form von Nahrungsmittelhilfe. Unter den NRO sind die wichtigsten Organisationen für die humanitäre Hilfe der DEZA : Caritas (über 9 Millionen), Terre des Hommes Lausanne (rund 5 Millionen), das Hilfswerk der Evangelischen Kirchen der Schweiz (rund 2 Millionen) und Ärzte ohne Grenzen (rund 2 Millionen).

Die Nahrungmittelhilfe bleibt relativ unverändert und geht im Verlauf der Zeit vielleicht sogar etwas zurück (32,3 Millionen Franken 1998 gegenüber 31,7 Millionen 1999). Sie besteht aus Milchprodukten (Milchpulver und Schmelzkäse) sowie Getreide (Reis, Mais, Weizen und Mehl). 1999 hat der Bund 2670 Tonnen an Milchprodukten für einen Betrag von 17,3 Millionen Franken sowie 27'571 Tonnen Getreide für einen Betrag von 14,4 Millionen Franken geliefert. Die Nahrungsmittelhilfe der Schweiz läuft über das Welternährungsprogramm (1999: 19,7 Millionen Franken) und über die schweizerischen Hilfswerke (1999: 11,8 Millionen), welche die Hilfe anschliessend verteilen.

\section{$\square$ Geographische Aufteilung der humanitären Hilfe 1999}

Geographisch gingen die Aufwendungen der Schweiz für humanitäre Hilfe 1999 vor allem in das Gebiet des früheren Jugoslawien. In finanzieller Hinsicht ist das humanitäre Programm in dieser Region das grösste, das jemals vom Bund durchgeführt wurde.

1999 hat sich die humanitäre Hilfe für Ex-Jugoslawien im Vergleich zu den Jahren 1997 und 1998 verfünffacht, vor allem wegen des Kriegs im Kosovo von März bis Juni 1999. In Albanien hat die Nothilfe und die Flüchtlingshilfe 1999 das Zehnfache der Leistungen für 1998 erfordert, die sich ihrerseits zwischen 1997 und 1998 bereits mehr als verdreifacht hatten. In diese beiden Länder flossen 1999 über 29\% der Aufwendungen der DEZA für die humanitäre Hilfe (einschliesslich der geographisch nicht aufgeschlüsselten Hilfe und der multilateralen Beiträge).

1999 war Afrika das zweitwichtigste Einsatzgebiet der schweizerischen humanitären Hilfe. Der afrikanische Kontinent erhielt fast $20 \%$ der gesamten Aufwendungen der DEZA für humanitäre Hilfe, in mehr als dreissig Ländern ver- 
teilt. Die Regionalprojekte für humanitäre Hilfe waren 1999 in dieser Region unbedeutend, während sie 1998 noch 1,5 Millionen Franken und 1997 das Doppelte erreicht hatten.

In Asien und im Mittleren Osten machten die Aufwendungen der DEZA für humanitäre Hilfe weniger als $12 \%$ des Gesamtbetrags aus, in über zwanzig Ländern verteilt; in Lateinamerika erreichten sie $5 \%$.

Tabelle 24: Aufwendungen der DEZA für die humanitäre Hilfe, nach Gebieten

\begin{tabular}{lrrr}
\hline & $\mathbf{1 9 9 9}$ & $\mathbf{1 9 9 8}$ & $\mathbf{1 9 9 7}$ \\
\hline Europa & 95532 & 17295 & 18476 \\
davon: Ex-Jugoslawien & 72510 & 13767 & 13208 \\
Albanien & 15670 & 1509 & 439 \\
\hline Afrika & 59364 & 48188 & 46809 \\
davon: Angola & 8642 & 7398 & 8297 \\
Sudan & 7433 & 3914 & 3032 \\
Ruanda & 6996 & 4681 & 4458 \\
Kongo (D.R.) & 5419 & 2749 & 2294 \\
Sierra Leone & 4329 & 2723 & 1200 \\
\hline Asien und Mittlerer Osten & 35102 & 28863 & 29872 \\
davon: Irak & 4629 & 2129 & 2541 \\
Georgien & 4549 & 3819 & 3868 \\
Georgien & 3883 & 4304 & 2700 \\
\hline Lateinamerika & 15449 & 12375 & 7862 \\
davon: Mittelamerika & 5584 & 3096 & 940 \\
Kuba & 2059 & 656 & 151 \\
Haiti & 579 & 2298 & 849 \\
Kolumbien & 1549 & 1014 & 1079 \\
\hline Geographisch nicht aufgeteilt & 76479 & 74296 & 74489 \\
davon: ordentliche Beiträge an das IKRK & 66000 & 65300 & 65000 \\
\hline Multilaterale Beiträge (ordentliche Beiträge) & 21846 & 43069 & 44604 \\
davon: UNHCR & 13000 & 13000 & 13000 \\
UNRWA & 7800 & 5000 & 7300 \\
IOM & 1009 & 1200 & 1200 \\
\hline Gesamtbetrag & $\mathbf{3 0 3 7 7 2}$ & $\mathbf{2 2 4 0 8 5}$ & $\mathbf{2 2 2 1 1 1}$ \\
\hline
\end{tabular}

Quelle: DEZA/seco. Internationale Zusammenarbeit der Schweiz, Jahresbericht 1999.

\section{$\square$ Humanitäre Hilfeleistungen der übrigen eidgenössischen Departemente}

1999 haben die übrigen eidgenössischen Departemente mehr als 90 Millionen Franken an humanitärer Hilfe aufgebracht. Zwei Drittel dieser Summe wurden vom Bundesamt für Flüchtlinge (BFF) im eidgenössischen Departement des Innern (EDI), hauptsächlich für die Aufnahme von Flüchtlingen aus Ex-Jugoslawien in der Schweiz und für die Hilfe zur Rückkehr dieser Flüchtlinge, erbracht. Das restliche Drittel erhielt der Kosovo vom Generalstab der Schweizer Armee (15 Millionen) und vom Heer (15 Millionen), die beide dem eidgenössischen Departement für Verteidigung, Bevölkerungsschutz und Sport (VBS) angehören.

\section{$\square$ Humanitäre Hilfeleistungen der Kantone und Gemeinden}

1999 haben die Kantone und Gemeinden etwas über 4 Millionen Franken für humanitäre Hilfe aufgebracht. Nahezu drei Viertel dieser Summe gingen nach Europa, davon 1,9 Millionen Franken in den Kosovo (Konfliktbewältigung) und 
930'000 Franken in die Türkei (Erdbebenhilfe). Lateinamerika hat von den Kantonen und Gemeinden insgesamt rund 340'000 Franken an humanitärer Hilfe erhalten, Afrika 170'000 Franken und Asien rund 150'000 Franken (Quelle: Statistischer Dienst der DEZA, Dezember 2000).

\subsection{WICHTIGSTE HUMANITÄRE AKTIONEN DER SCHWEIZ IM JAHR 2000}

Die DEZA hat im Jahr 2000263 Millionen Franken an humanitärer Hilfe aufgebracht. Unter allen humanitären Aktionen der DEZA waren die Einsätze im Kosovo, in Afghanistan und in Mosambik am bedeutendsten.

\section{$\square$ Kosovo}

Auch im Jahr 2000 war der Kosovo der Hauptempfänger der humanitären Hilfe der Schweiz. Der für humanitäre Einsätze in dieser Region bereitgestellte Kredit betrug über 40 Millionen Franken.

Die von der Schweiz, Russland, Griechenland und Österreich gemeinsam durchgeführte Initiative «Focus» wurde im Februar 2000 abgeschlossen. Sie war im Frühjahr 1999 eingeleitet worden, als die humanitären Organisationen gezwungen waren, ihre Aktivitäten in der Region nach den NATO-Bombardierungen fast völlig einzustellen. Gemäss der DEZA ist die Bilanz positiv ${ }^{3}$ : die Nothilfeoperation habe es erlaubt, die ersten Ansätze für künftige bilaterale Projekte festzulegen und nützliche Erfahrungsgrundlagen im Bereich der humanitären Hilfe zu gewinnen.

Die Rückkehrhilfe für die Kosovo-Flüchtlinge, die während des Krieges in der Schweiz aufgenommen wurden, wird zu 80\% vom Bundesamt für Flüchtlinge (BFF) finanziert (siehe JSDW 2001, Kapitel 5.3.1., Punkt 3), obwohl die DEZA im Kosovo für das Programm verantwortlich ist. Das Programm umfasst unter anderem den Bau und die Renovierung von 700 Häusern und einer Reihe Schulen für mehr als 3500 Studierende. Ferner fördert die humanitäre Hilfe der Schweiz die Wiederinstandsetzung des Trinkwassernetzes für eine Bevölkerung von 150'000 Einwohnern im Westen des Kosovo. Die Unterstützung der Landwirtschaft wurde im Jahr 2000 mit der Entsendung von weiteren 1000 Stück Vieh fortgesetzt, womit sich der Gesamtbestand an Schweizer Kühen in der Provinz Kosovo (einschliesslich Neugeburten vor Ort) auf über 2000 beläuft. Ferner wurden Saatkartoffeln geliefert.

Schliesslich beteiligt sich die Schweiz in Zusammenarbeit mit dem russischen Zivilschutz Emercom an der Minenräumung im Kosovo. Das eidgenössische Departement für Verteidigung, Bevölkerungsschutz und Sport (VBS) stellt in diesem Bereich im Kosovo und in Bosnien-Herzegowina eine schweizerische Technologie bereit, welche die Entschärfung bzw. Sprengung von Blindgängern und Minen ohne manuelle Berührung erlaubt (8600 Einheiten werden in beiden Gebieten geliefert). Im Kosovo arbeitet das VBS in Partnerschaft mit einer deutschen Nichtregierungsorganisation, Help, und mit dem Minenräumzentrum der UNO, das die Räumaktionen in der Region koordiniert.

3. DEZA, Humanitäre Initiative «Focus»: Positive Bilanz der vier Trägerstaaten, Pressemitteilung vom 14. Februar 2000. 


\section{$\square$ Afghanistan}

Die Schweiz hat die (turnusmässig wechselnde) Präsidentschaft der Gruppe zur Unterstützung Afghanistans (Afghanistan Support Group - ASG, 1996 von der UNO gegründet) für das ganze Jahr 2000 übernommen und wird den Vorsitz 2001 an Deutschland übergeben. Die Mitglieder dieser Gruppe (15 Länder und die Europäische Union) sowie eine Reihe humanitärer UN- und Nichtregierungsorganisationen haben sich am 7. und 8. Dezember 2000 zur siebten ASGKonferenz in Montreux getroffen. Ihre wichtigste Botschaft war für den Sicherheitsrat der Vereinten Nationen bestimmt. Er wird aufgefordert, keine Verstärkung der Sanktionen gegenüber dem Taliban-Regime zu beschliessen, da solche Sanktionen ihres Erachtens katastrophale humanitäre Folgen hätten, indem sie die Abreise der vor Ort tätigen humanitären Organisationen erzwingen würden. Die DEZA hat im Jahr 200010 Millionen Franken für Afghanistan, hauptsächlich zugunsten der Flüchtlinge und Vertriebenen, aufgebracht. Neben der Lieferung von Nahrungsmitteln und Medikamenten hat sich die DEZA vor allem am Wiederaufbau vom Krieg zerstörter Dörfer beteiligt. Afghanistan leidet aber seit 1999 zusätzlich zur Kriegssituation auch an einer Dürre. Vor Ort ist eine gross angelegte Partnerschaft mit dem IKRK, dem UN-Flüchtlingshilfswerk, der Internationalen Organisation für Migration, dem Welternährungsprogramm, der FAO und der UN-Organisation Habitat im Einsatz, um nur die Wichtigsten zu nennen.

\section{$\square$ Mosambik}

Die schweren Überschwemmungen, die Mosambik im Februar 2000 heimsuchten, haben die DEZA zur Freigabe einer Nothilfe von 5 Millionen Franken veranlasst. Hinzu kam noch ein gleich hoher Betrag für technische Hilfe, da Mosambik ein Schwerpunktland der DEZA ist. Ende März 2000 hat das seco zusätzlich eine nicht rückzahlbare Soforthilfe an Mosambiks Zahlungsbilanz in Höhe von 5 Millionen Franken geleistet.

Die Nothilfe erfolgte in Form von Material (Decken, Wassereimer, Medikamenten) und Hilfsnahrungsmitteln, ferner durch Fachpersonal (Ärzte und Logistiker) sowie durch die Finanzierung der Einsätze der internationalen Organisationen vor Ort, darunter das Welternährungsprogramm (WEP), das die Evakuierung der Flutopfer mit Hubschraubern durchführte.

Die DEZA hat auch einen Beitrag zum Wasser- und Hygieneprogramm der UNICEF (Water and Sanitation Programme) geleistet. Ferner hat sie das mosambikanische Gesundheitsministerium und die nationale Trinkwasseragentur unterstützt.

\section{$\square$ Weitere humanitäre Aktionen}

Im Juni 2000 hat die DEZA 4 Millionen Franken (die Hälfte davon für die Einsätze des WEP) zugunsten Nordkoreas bereitgestellt, das aufgrund seiner wirtschaftlichen Isolierung an chronischem Getreidemangel leidet.

Das Horn von Afrika und der Sudan, die unter der Dürre leiden, erhielten im Rahmen der humanitären Hilfe der Schweiz 15 Millionen Franken, davon 4,5 Millionen Franken für Äthiopien. Vor Ort verwaltet vor allem das WEP die Verteilung der Hilfsnahrungsmittel (einschliesslich des Unterhalts und der Instandsetzung des dafür notwendigen Strassennetzes). 
Die Überschwemmungen des Mekongs, welche Vietnam, Kambodscha und Laos im September 2000 heimsuchten, wie auch die Überschwemmungen in Bangladesch und Indien haben die DEZA veranlasst, rund eine Million Franken an humanitärer Hilfe zur Verfügung zu stellen. Die DEZA ist bereits mit ihren Koordinationsbüros in Hanoi, Dhaka und Delhi präsent und hat die Hilfe auf die Unterstützung ihrer Partner (vor allem Caritas, Schweizerisches Rotes Kreuz und Internationale Föderation der Rotkreuz- und Rothalbmondgesellschaften) konzentriert, die unter anderem Nahrungsmittel, Medikamente und Kleidung an die Flutopfer verteilten.

Im März 2000 hat die DEZA 3,5 Millionen Franken für das IKRK, das UNHCR und das WEP für ihre Aktion in Dagestan, Inguschien und Nordossetien bereitgestellt, wo rund 200'000 durch den Krieg vertriebene Tschetschenen Zuflucht gefunden haben. In Tschetschenien selbst sollen weitere 300'000 Personen vertrieben worden sein.

Im Dezember 2000 hat die Schweiz eine Million Franken an humanitärer Hilfe für die Opfer der Feindseligkeiten im Gazastreifen und im Westjordanland bereitgestellt. Dieser Betrag kommt zu den 150'000 Franken hinzu, die von der Schweiz schon zu Beginn der israelisch-palästinensischen Gewalttätigkeiten im Oktober 2000 freigegeben worden waren. Die Summe von einer Million Franken ist Teil einer internationalen Verpflichtung von 39 Millionen Franken, die dem Hilfswerk der Vereinten Nationen für die Pälestinaflüchtlinge im Nahen Osten (UNRWA) zur Verfügung gestellt werden. Zudem hat der Bundesrat ebenfalls im Dezember beschlossen, das medizinische Nothilfeprogramm des IKRK in dieser Region mit 1,5 Millionen Franken (Zusammenarbeit mit dem palästinensischen Roten Halbmond) zu unterstützen.

Die DEZA hat auch die Expertenmissionen der UNO unterstützt, die nach der Verschmutzung der Donau und der Theiss mit Zyanid und Schwermetallen in Ungarn und Rumänien im Einsatz waren.

Am 29. März 2000 hat der Bundesrat einen neuen Kredit von 42 Millionen Franken für das Welternährungsprogramm (WEP) für die Jahre 2000 und 2001 beschlossen. Damit wird sich die Schweiz an den Aktionen des WEP mit Barzahlungen und durch Nahrungsmittelhilfe in Form von Milchprodukten beteiligen.

Im Oktober 2000 hat der Bundesrat einen Nachtragskredit von 20 Millionen Franken für das IKRK bewilligt, womit sich der Beitrag der Schweiz an das Budget des IKRK auf 100 Millionen Franken erhöht. Dieser Nachtragskredit «dient der Weiterführung der deutlich unterfinanzierten Hilfsprogramme des IKRK ${ }^{4}$ in verschiedenen Ländern, da die stetig wachsende Zahl von Krisen und Konflikten in der Welt die Finanzierung dieser Programme immer schwieriger macht. Schliesslich sei der Anekdote halber noch erwähnt, dass die Schweiz Nothilfe an Frankreich (Lieferung von Notstromaggregaten) geleistet hat, als im Januar 2000 nach dem Wirbelsturm «Lothar» eine Million Menschen ohne Stromversorgung waren.

4. EDA, IKRK erhält zusätzlich 20 Millionen Franken von der Schweiz, Pressemitteilung vom 25. Oktober 2000 . 


\subsection{INTERNATIONAL BEDEUTENDE EREIGNISSE ZUM THEMA HUMANITÄRE HILFE}

\section{$\square$ Zentrum Henry Dunant für den humanitären Dialog}

Der Bundesrat hat dem Parlament am 31. Mai 2000 seine «Botschaft über die Teilnahme und den finanziellen Beitrag des Bundes an das Zentrum Henry Dunant für den humanitären Dialog für die Jahre 2001 bis $2003 »^{5}$ unterbreitet. Nach der Unterstützung der Aussenpolitischen Kommission des Ständerates am 24. August 2000 haben die eidgenössischen Räte am 18. September 2000 das Gesetz und den einfachen Bundesbeschluss verabschiedet, für welche die Botschaft des Bundesrates eingetreten war. Der Bund wird sich somit am Zentrum Henry Dunant für den humanitären Dialog beteiligen, dessen Vertreter im Stiftungsrat der Staatssekretär des EDA sein wird. Die Beiträge des Bundes an das Zentrum Henry Dunant sind auf insgesamt 2'850'000 Franken für die Jahre 2001 bis 2003, das heisst auf höchstens 950'000 Franken pro Jahr, festgesetzt.

Das Zentrum Henry Dunant, welches das Institut Henry Dunant im November 1998 abgelöst hat, ist eine privatrechtliche Stiftung unter Aufsicht des Bundes. Es hat zur Aufgabe, einen interdisziplinären, multikulturellen und universellen Dialog zu fördern, mit dem Ziel, nachhaltige Lösungen für die Probleme der humanitären Aktion im Feld zu finden. Besonders soll die Akzeptanz der humanitären Prinzipien bei allen an Konfliktsituationen beteiligten staatlichen und nichtstaatlichen Akteuren gesteigert werden.

Das Anfangskapital des Zentrums wurde vom IKRK, von der Internationalen Föderation der Rotkreuz- und Rothalbmondgesellschaften und vom Schweizerischen Roten Kreuz vollständig gezeichnet. Die UNO und der Bund haben beide einen ständigen Sitz im Stiftungsrat, dessen Zusammensetzung die Aktivitätsbereiche und die geographische Vielfalt der von humanitären Fragen betroffenen Akteure widerspiegelt. Die Arbeit des Zentrums Henry Dunant soll die Tätigkeit der im Feld aktiven internationalen humanitären Organisationen ergänzen.

\section{$\square$ Vom OCHA organisiertes Freiburger Forum}

Das UN-Büro für die Koordination humanitärer Angelegenheiten (OCHA) hat am 15. und 16. Juni 2000 eine internationale Ministerkonferenz über Krisenbewältigung und regionale Zusammenarbeit in Freiburg organisiert. Vertreter aus 51 Ländern und von 37 regionalen Organisationen nahmen daran teil. Das Ziel der Konferenz war, einen neuen politischen Rahmen zu schaffen, um die Wirksamkeit und die Kohärenz der humanitären Reaktion auf Notsituationen und Natur- oder industrielle Katastrophen zu gewährleisten. Die parallel laufenden Einsätze und die mangelnde Koordination führen zur Verschwendung der für die humanitäre Hilfe eingesetzten Mittel, wobei die vom Freiburger Forum beschlossenen Massnahmen und Empfehlungen eben darauf abzielen, gegen diese Vergeudung anzugehen. Die Umsetzung dieser Massnahmen und Empfehlungen kommt langsam in Gang.

5. Bundesrat, «Botschaft über die Teilnahme und den finanziellen Beitrag des Bundes an das Zentrum Henry Dunant für den humanitären Dialog für die Jahre 2001-2003» vom 31. Mai 2000 (Botschaft Nr. 00.043). 


\section{$\square$ Drittes Zusatzprotokoll zu den Genfer Abkommen}

Im September 2000 hat die Schweiz beschlossen, eine diplomatische Konferenz für den 25. und 26. Oktober 2000 in Genf, im Hinblick auf die Verabschiedung eines drittes Zusatzprotokolls zu den Genfer Abkommen über das Emblem einzuberufen. Dieser Beschluss folgte den Konsultationsgesprächen vom 5. und 6. September 2000, bei denen rund hundert Vertragsstaaten der Genfer Konventionen ihre Ansichten über die Vorschläge des IKRK bezüglich der Einführung eines neuen Emblems austauschen konnten. Dieses neue Wahrzeichen würde es insbesondere der israelischen Gesellschaft Magen David Adom erlauben, sich der Internationalen Bewegung der Rotkreuz- und Rothalbmondgesellschaften anzuschliessen, aus der sie wegen der Emblemfrage seit mehreren Jahrzehnten ausgeschlossen wurde.

Die Ereignisse der Intifida der Moscheen seit Oktober 2000 haben die Konkretisierung der bis dahin erreichten diplomatischen Fortschritte jedoch unmöglich gemacht. Daher hat die Schweiz die Verschiebung der diplomatischen Konferenz auf Anfang des Jahres 2001 angekündigt.

\section{QUELLEN}

DEZA, Internationale Zusammenarbeit der Schweiz. Jahresbericht 1999. Humanitäre Hilfe, $16 \mathrm{~S}$.

DEZA, Eine Welt, Nr. 4, Dezember 2000 (mit Artikelreihe über den Balkan), $35 \mathrm{~S}$.

DEZA, DEZA-Hilfe für Mekong-Flutopfer in Südostasien, Pressemitteilung vom 22. September 2000.

DEZA, Humanitäre Initiative « Focus »: Positive Bilanz der vier Trägerstaaten, Pressemitteilung vom 14. Februar 2000.

DEZA, DEZA verstärkt Hilfe für Flutopfer in Südasien, Pressemitteilung vom 27. September 2000.

DEZA, Erhöhung der Nothilfe der Schweiz für die Gewaltopfer in Palästina, Pressemitteilung vom 19. Dezember 2000.

EDA, Schweiz übernimmt das Präsidium der «Afghanistan Support Group» für das Jahr 2000, Pressemitteilung vom 10. Dezember 1999.

EDA, IKRK erhält zusätzlich 20 Millionen Franken von der Schweiz, Pressemitteilung vom 22. Oktober 2000.

VBS, Schweizer Entminungssystem für den Balkan, Pressemitteilung vom 4. April 2000.

Le Temps, Trois Suisses en éclaireurs dans Grozny dévasté, 14. März 2000.

Le Courrier, Les Coréens du Nord ont toujours faim, 14. Juni 2000.

OCHA, Le Fribourg Forum sur la gestion des crises abordera des questions épineuses: la lutte contre des doubles emplois et le gaspillage de fonds dans l'action humanitaire, Communiqué de presse du 7 juin 2000.

seco, Weitere Wiederaufbauhilfe der Schweiz nach den verheerenden Überschwemmungen in Mosambik, Pressemitteilung vom 30. März 2000.

\section{INTERNET-ADRESSEN}

DEZA: www.deza.admin.ch

SKH: www.skh.ch

OCHA : www.reliefweb.int/ocha

IKRK : www.icrc.org 